\title{
SEARCH FOR SUPERLUMINAL MOTION IN THE WEAK CORES OF EXTENDED QUASARS
}

\author{
J. A. Zensus \\ California Institute of Technology, Pasadena, U.S.A. \\ R. W. Porcas \\ Max-Planck-Institut für Radioastronomie, Bonn, F.R.G.
}

\begin{abstract}
We present a status report on a project to study structural changes in a sample of radio cores associated with quasars with extended double lobes. Of six objects observed, so far two (3C 179 and 3C 263) are superluminal and two more (3C 268.4 and $1951+49)$ are possible superluminal candidates.
\end{abstract}

The search for superluminal motion in the weak cores of double radio sources provides a critical test of simple beaming models commonly used to explain the statistics of superluminal sources (Scheuer and Readhead, 1979; Blandford and Königl, 1979; Orr and Browne, 1982). It follows from the requirement of a narrow relativistic jet oriented close to the line-of-sight to the observer that the superluminal effect should be rare in a complete sample of sources selected on the basis of the strength of the extended emission, i.e. without orientation bias due to the central components. Such a sample of sources is provided by the 30 quasars from the Jodrell Bank $966 \mathrm{MHz}$ survey that were mapped with arcsecond resolution by Owen et al. $1978\left(\mathrm{~S}_{966} \geq 0.7 \mathrm{Jy}, \mathrm{m}_{b} \leq 19 \mathrm{mag}\right.$, and angular size $>10$ arcsec). We have continued our project to study the milli-arcsecond structures and

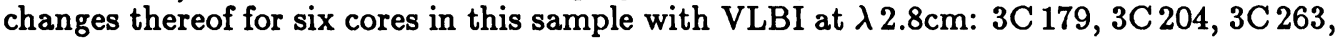
3C 268.4, 1732+65, 1951+49 (see Zensus and Porcas, 1984, for a list of source properties). These cores range in flux density between 50 and $300 \mathrm{mJy}$ and are therefore ca. 100 times weaker than the core-dominated superluminal sources. The measurement of superluminal expansion in $3 \mathrm{C} 179$ with a speed of ca. $10 c\left(H_{0}=55 \mathrm{~km} \mathrm{~s}^{-1} \mathrm{Mpc}^{-1}\right.$ and $\left.q_{0}=0.5\right)$ is well established and reported elsewhere (Porcas, 1981, and Porcas, these proceedings). Here we concentrate on the results for the other sources, except 3C 204, which was not detected in our observations. Fig. 1a-c show hybrid maps for 3C 263, 3C 268.4, and 1951+49 (a model fit to the data of $1732+65$ shows a compact core, possibly double, extended roughly along the overall source axis). The structural properties of these objects can be summarized as follows:

a. These cores have simple double structures accounting for large fractions of the total core fluxes and similar to the structures found in core-dominated objects. 3C 179 can be considered a prototype for this class of object.

b. Despite the lack of spectral information for the components their morphology is suggestive of a "core-jet" structure.

c. An "orientation memory" is found between features on pc- and kpc-scales. Bending of the structures seems to occur not within the compact regions but rather through interaction of the jets with the external medium. 
STRUCTURAL CHANGES: All quasars repeatedly observed show structural changes. From 3 observing epochs for 3C 263 superluminal expansion at a speed of ca. 3c was derived (Zensus, Hough and Porcas, 1986). This is the weakest superluminal source found so far $\left(S_{\lambda 2.8} \sim 150 \mathrm{mJy}\right)$. Preliminary results from second epoch data for 3C 268.4 and $1951+49$ suggest that both are possible superluminal candidates, too.

CONCLUSION: Our results indicate that superluminal motion is a common phenomenon among the weak cores of extended quasars, in contradiction to expectations based on simple versions of the beaming hypothesis.

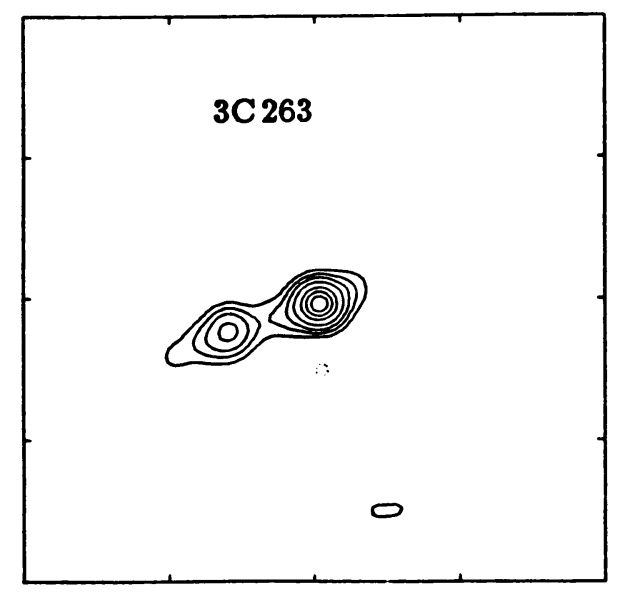

FIGURE 1 :

VLBI-maps $(\lambda 2.8 \mathrm{~cm})$ of cores in $3 \mathrm{C} 263$, $3 \mathrm{C} 268.4$, and $1951+49$ (scale 1 milliarcsecond per tick; size of restoring beam $0.2 \times 0.2$ m.a.s.).
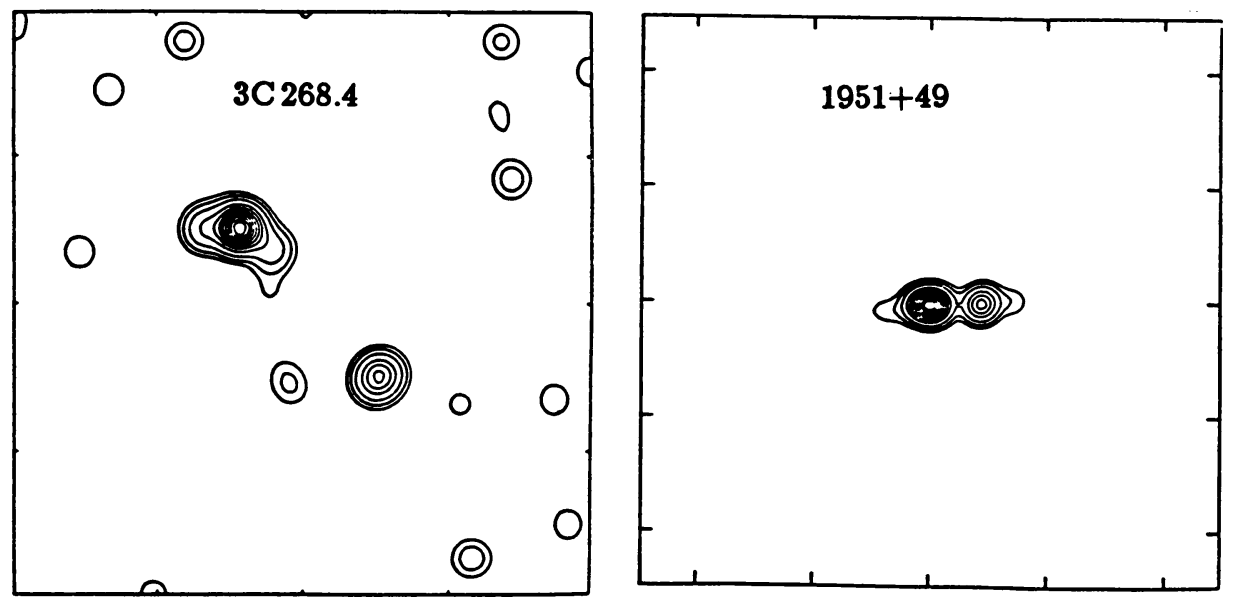

\section{REFERENCES}

Blandford, R. D. and Königl, A.: 1979, Astrophys. J. 232, 34

Orr, M. J. L., Browne, I. W. A.: 1982, Monthly Not. Roy. Astron. Soc. 200, 1067

Owen, F. N., Porcas, R. W., Neff, S. G.: 1978, Astron. J. 83, 1009

Porcas, R. W.: 1981, Nature 294, 47

Scheuer, P. A. G. and Readhead, A. C. S.: 1979, Nature 277, 182

Zensus, J. A. and Porcas R. W.: 1984, proc. IAU Symp. 110, 163

Zensus, J. A., Hough, D. H., Porcas, R. W.: 1986, in preparation 